\title{
Students' Satisfaction and Performance in Flipped Physics Classrooms Having Different Proportions of Face-to-face Lecturing
}

\author{
Heeseung ZoE \\ School of Undergraduate Studies, College of Transdisciplinary Studies, DGIST, Daegu 42988, Korea \\ Department of Physics Education, Pusan National University, Busan 46241, Korea \\ Research Center for Dielectric and Advanced Matter Physics, \\ Pusan National University, Busan 46241, Korea \\ Department of Physics Engineering, Istanbul Technical University, Maslak 34469, Turkey \\ Kyujin KWAK \\ Department of Physics, UNIST, Ulsan 44919, Korea

\section{Dong-han YEOM} \\ Department of Physics Education, Pusan National University, Busan 46241, Korea \\ Research Center for Dielectric and Advanced Matter Physics, \\ Pusan National University, Busan 46241, Korea \\ Jihoon LEE* \\ School of Undergraduate Studies, College of Transdisciplinary Studies, DGIST, Daegu 42988, Korea
}

(Received 25 September 2020 : revised 3 December 2020 : accepted 4 December 2020)

Flipped learning is an instructional design implementing peer instruction by reducing traditional face-to-face lecturing. However, students tend to be afraid of replacing face-to-face lecturing with peer instruction. We applied the flipped learning method to two sections of General Physics 2 for electromagnetism and modern physics. We controlled the ratio of face-to-face lectures to the entire class meeting hours to be $1 / 2$ for one section $(\mathrm{N}=288)$ and $1 / 3$ for the other section $(\mathrm{N}=296)$. Students' achievements were not statistically distinguished between the two sections. Students' satisfaction with lecturing was indistinguishable. However, while students in the $1 / 3$ section prefered peer instruction, those in the $1 / 2$ section were satisfied with instructional design. According to a correlation analysis, students' satisfaction correlated with achievements only in the $1 / 2$ section. Therefore, we conclude that flipped learning is systematically worked in the $1 / 2$ section rather than in the $1 / 3$ section.

Keywords: Physics education, Flipped learning, Peer instruction

\section{플립트 러닝이 적용된 물리 수업에서 면대면 강의의 분량과 수강생의 만족도 및 성취도의 관계

\author{
조희승
} \\ DGIST 융복합대학 기초학부, 대구 42988 , 대한민국}

This is an Open Access article distributed under the terms of the Creative Commons Attribution Non-Commercial License (http://creativecommons.org/licenses/by-nc/3.0) which permits unrestricted non-commercial use, distribution, and reproduction in any medium, provided the original work is properly cited. 
부산대학교 물리교육과, 부산 46241 , 대한민국

부산대학교 유전체물성연구소, 부산 46241 , 대한민국

이스탄불 공과대학교 물리공학과, 이스탄불 34469 , 터키

\section{곽규진}

UNIST 물리학과, 울산 44919, 대한민국

\section{염동한}

부산대학교 물리교육과, 부산 46241 , 대한민국 부산대학교 유전체물성연구소, 부산 46241 , 대한민국

\section{이지훈}

DGIST 융복합대학 기초학부, 대구 42988 , 대한민국

(2020년 9월 25 일 받음, 2020 년 12 월 3 일 수정본 받음, 2020 년 12 월 4 일 게재 확정)

플립트 러닝은 전통적인 면대면 강의의 비중을 줄이고 동료 학습을 통해 효과적인 학습을 달성하는 방법이다. 하지만 동료 학습이 면대면 강의를 대체하는 것에 대해 수강생들은 어려움을 느낀다. 우리는 일반물리 2 수업 2 개 분반에 플립트 러닝을 적용하여 수강생들의 학업 성취도와 수업 만족도를 평가했다. 두 학기 동안 한 분반은 면대면 강의의 분량이 전체 수업 시간의 $1 / 2$ 이 되게 하고, 다른 한 분반은 $1 / 3$ 이 되도록 조절하고, 나머지는 문제풀이 기반의 동료 학습을 시행했다. 학업 성취도는 두 분반에서 통계적으로 동일했다. 면대면 강의에 대한 만족도는 분반 사이에 큰 차이가 없었으나, 동료 학습 만족도는 $1 / 3$ 분반이, 수업 설계 만족도는 $1 / 2$ 분반이 높은 것으로 나타났다. 상관분석의 결과는 $1 / 3$ 분반은 그렇지 않으나 $1 / 2$ 분반은 수업 만족도와 학업 성취도 사이에 유의한 상관관계가 있 는 것으로 나타났다. 따라서 $1 / 3$ 분반 보다는 $1 / 2$ 분반에 플립트 러닝이 체계적으로 동작하는 것으로 보인다.

Keywords: 물리교육, 플립트 러닝, 동료 학습

\section{I. 서 론}

플립트 러닝 (flipped learning) [1,2] 은 학습자가 스스로 수업을 준비하여 강의실 수업에서 교수자와 상호작용을 극대화하는 방법으로서, 대학 수준의 물리학을 가르치는 데에도 자주 사용하고 있다 $[3,4]$. 플립트 러닝의 기본 구 조는 [3]에서 언급한 바와 같이 수업 시간 내에 교수자가 학습자에게 즉각적으로 피드백을 주는 "시의적 학습 (justin-time-teaching)" [5] 과 학습자들이 협업하여 서로의 이 해를 돕는 "동료 학습(peer instruction)" [6,7]을 병행하는 형태이다. 일반적으로 플립트 러닝을 적용한 교실에서 교수 자는 수업을 시작하기 전에 학습자에게 컨텐츠를 제공하여 스스로 예습하게 한 후, 수업 시간에는 교수자가 일방적으로 강의하는 대신, 학습자가 능동적으로 컨텐츠를 체화할 수 있도록 동료들과 대화형 수업을 진행한다.

플립트 러닝은 다음과 같은 두 가지 맥락에서 그 필요성이 분명해진다. 첫째, 플립트 러닝에 활용할 수 있는 개방형

*E-mail: vastlee4@dgist.ac.kr
온라인 교육 자료들이 활발하게 개발되고 있다. 전 세계 선 도적인 대학들은 MOOCs(Massive Open Online Courses) 라는 이름으로 양질의 강의들을 웹에 공개하고 있다. 플 립트 러닝의 구조 안에서 교수자는 이를 학습자의 예습을 위한 자료로 적절하게 활용할 수 있다 [8-10]. 둘째, 교수 자의 일방적인 강의 대신 교수자와 학습자의 상호작용을 이끌어내는 효과적인 교육 기법들이 연구되었다. 적어도 물리 교육에 있어서는 학습자들이 소그룹 문제 풀이와 같은 협업적 교육을 경험할 때 $[11,12]$, 그리고 교수자와 학습자 혹은 학습자들 간에 연계성 (engagement)을 형성할 때 $[6$, 7,13-16], 교과 내용을 보다 효과적으로 배울 수 있다는 것 이 분명해졌다. 플립트 러닝의 틀 안에서 교수자의 목표와 학습자의 수준에 따라 이러한 효과적인 기법들을 적절하게 선별하여 사용할 수 있다.

플립트 러닝은 전통적인 강의 중심의 수업에 비해 학습 자가 자유롭게 배움의 과정을 조절하고 선택할 수 있다 [1]. 전통적인 면대면 강의 위주의 수업에서는 교수자가 분명한 교육 목표를 전달하고 학습 컨텐츠의 범위를 획일적으로 정한다. 하지만 플립트 러닝에서는 수업에 들어 오기 전에 
학습자가 수업 내용을 준비하는 수준을 스스로 결정하고 교육적 목표를 설정한다. 수업 시간에는 자신이 준비한 것 을 기반으로 수업에 참여하여 이 목표를 달성한다. 하지만 바로 이런 면이 잠재적으로 플립트 러닝의 불안 요소가 되기 도 한다. 만약 학생들이 능동적으로 학습에 대해 준비하고 수업에 들어오지 않는다면, 의미있는 학습이 이뤄지기 어렵 다. 이런 맥락에서 플립트 러닝의 구조 안에 전통적 면대면 강의로 학습자의 준비 정도에 상관 없이 코스의 전반적인 목표가 이뤄지도록 안전 장치를 마련할 필요가 있다. 그러 므로 학습자의 예습, 연계성을 강화하는 교육 기법, 그리고 전통적 면대면 강의 요소가 결합된 형태의 플립트 러닝을 적용할 때 면대면 강의의 역할과 효용을 세심하게 연구할 필요가 있다. 이것은 플립트 러닝을 적용하여 교과목을 설 계하는 교수자들이 가질 수 있는 의문이며, 여러 가지 방향 으로 탐색해야 하는 연구 주제가 된다.

저자들의 선행연구 [4]에서 이미 U대학의 일반 물리 1(대 학 신입생들이 봄 학기에 주로 역학, 열역학을 미적분학 기 반으로 배우는 기초 물리 교과) 에 플립트 러닝을 적용하여 2 번의 학기에 걸쳐 연구를 진행했다. 이 연구에서는 면대면 강의 분량을 주요한 변인으로서 삼았다. 첫번째 학기에는 한 교수자는 200 명 규모의 분반에 전통적인 강의 중심의 수 업을 진행했고, 다른 한 교수자는 200 명 규모의 다른 분반에 플립트 러닝을 적용하여 수업을 진행했다. 고등학교에서 다룬 주제들과 겹치는 중간 고사까지 두 분반의 전체적인 성적 분포가 유사했으나 기말고사에서는 플립트 러닝을 적 용한 분반의 성적이 전통적 강의 중심의 분반의 성적보다 통계적으로 의미있게 높았다. 두번째 학기에는 한 교수자가 200 명 규모의 한 분반에는 면대면 강의 분량을 $1 / 2$ 로, 다른 한 분반에는 $1 / 3$ 로 하고 나머지 수업 시간은 소그룹 문제 풀이 중심의 대화형 강의를 진행했다. 중간고사와 기말고 사에서 두 분반의 학업 성취도는 통계적으로 차이가 없다는 것을 확인할 수 있었다. 그러므로 우리는 플립트 러닝을 효과적으로 설계하여 수업에 적용하면, 면대면 강의의 비 율이 줄어들어도 학습자들의 학습효과가 크게 떨어지지 않 는다는 것을 확인할 수 있었다. 하지만 만약 학습자들에게 다소 새롭고 복잡한 주제들이 주어졌을 때, 플립트 러닝이 효과적으로 동작할 수 있을까에 대한 고민이 본 연구를 진 행하게 된 동기이다.

본 논문은 $\mathrm{U}$ 대학에서 일반 물리 2(대학 신입생들이 가을 학기에 주로 전자기학, 현대 물리학을 미적분학 기반으로 배우는 기초 물리 교과) 에 플립트 러닝을 적용한 연구이다. 일반 물리 2 는 일반 물리 1 에 비해 학생들이 개념을 이해 하기 어렵고, 수학적 표현 너머에 있는 논리를 이해하는 것은 더욱 어렵다. 보통 학생들은 교수자가 면대면 강의로 이러한 주제들을 세심하게 설명해 주기를 기대한다. 그러나
Table 1. The Class Formats of Two Sections.

\begin{tabular}{c|c|c}
\hline \hline \multirow{2}{*}{ Before Class } & \multicolumn{2}{|c}{ Section } \\
\cline { 2 - 3 } & \multicolumn{2}{|c}{ Lecture notes, movie clips and } \\
& \multicolumn{2}{|c}{ Qeading materials are assigned. } \\
& Face-to-face lecture: & Face-to-face lecture: \\
\multirow{3}{*}{ In Class } & 90 minutes & 60 minutes \\
& Group discussion : & Group discussion : \\
& 90 minutes & 120 minutes \\
\hline After Class & \multicolumn{2}{|c|}{ Homework problems are assigned. } \\
\hline \hline
\end{tabular}

이것은 전통적 강의를 간소화하고 다양한 활동을 추구하는 플립트 러닝의 기본 방향과는 거리가 있다. 따라서 일반 물 리 2 에 플립트 러닝을 적용하였을 때 면대면 강의의 역할을 세심하게 살펴볼 필요가 있다.

본 연구는 2 번의 학기에 걸쳐 진행하였으며, 각 학기마다 같은 학력 수준의 2 개 분반에 면대면 강의 분량을 $1 / 2$ (이하 $1 / 2$ 분반) 과 $1 / 3$ (이하 $1 / 3$ 분반) 로 통제하였다. 수업 형식을 요약하면 Table 1 과 같다. 수업에 들어오기 전에 학생들은 교수자가 준비한 강의 노트, 영상, 읽기 자료들을 숙지하 고, 퀴즈 문제를 풀어야 한다. 교실 수업의 전체적인 구 성은 교수자가 내용을 전달하는 면대면 강의 (face-to-face lecturing) 와 학생들이 옆에 앉은 $2-3$ 명의 동료들과 진행 하는 동료 학습 기반의 토론 (group discussion)이다. 수업 은 일 주일에 2 회 진행하였고, 1 회는 90 분으로 구성되었 다. $1 / 2$ 분반은 매회 45 분은 면대면 강의에, 나머지 45 분은 토론식 문제 풀이에 활용하였다. 그러나 $1 / 3$ 분반은 첫 회 수업의 60 분은 면대면 강의에, 나머지 30 분은 토론식 문제 풀이에 활용하였다. 두번째 회의 수업은 토론식 문제 풀 이만으로 진행하였다. 한 주간의 모든 수업이 끝나면 연습 문제를 과제로 내주었으며, 학생들에게 개별적으로 그것을 풀어 제출하게 하였다. 본 논문의 연구 질문을 요약하면 다음과 같다.

- 면대면 강의의 분량은 학습자의 학업 성취도에 어떠 한 영향을 미치는가?

- 면대면 강의의 분량은 학습자의 만족도와는 어떠한 관계가 있는가?

\section{II. 방 법}

선행연구인 [3] U대학에서 플립트 러닝을 적용한 일반 물리 2 의 수업 구조와 전통적인 강의 중심의 수업 구조를 비교하면 Fig.1과 같다. 학생들은 수업에 들어 오기 전에 


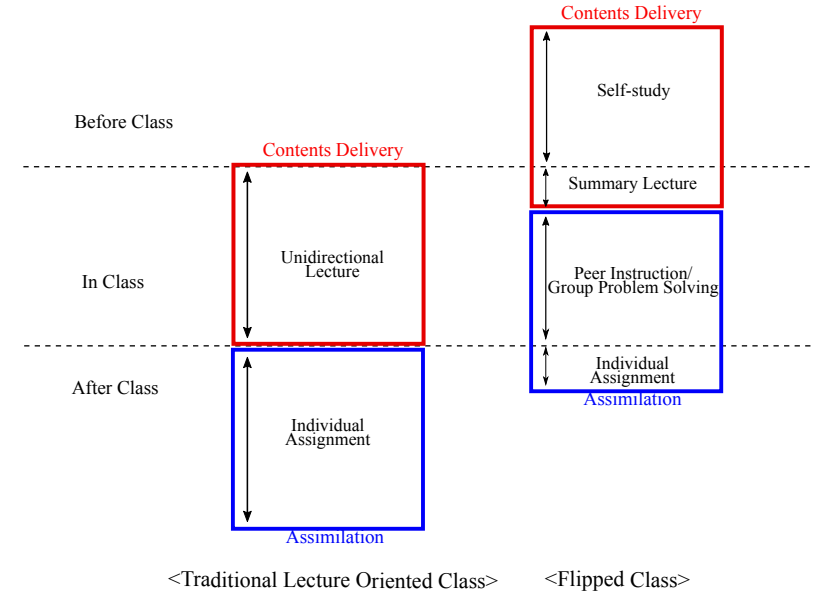

Fig. 1. (Color online) Comparision of the structures of traditional lecture oriented class and flipped class. (Credit: [4])

LMS(Learning Management System) 상에서 동영상 강의 와 강의 노트를 학습하고 객관식 문제로 구성된 퀴즈 문제를 풀어야 한다. 학생들은 퀴즈를 여러 번 반복해서 시도할 수 있었고, 정답률이 $80 \%$ 이상이 되는 결과만 성적에 산출하 기로 했다.

수업 시간에 교수자는 두 가지 형식의 강의를 준비한다. 먼저 예습으로 내어준 컨텐츠를 요약하고 중요한 쟁점을 다시 부각시켜 설명하는 전통적 면대면 강의를 진행한다. 이어서 학생들을 $3-5$ 명 정도의 소그룹으로 나누어 문제 풀이 중심의 동료 학습을 진행한다 $[5-7,11,12]$. 교수자는 컨텐츠의 핵심 주제를 반영하는 문제를 엄선하여 학생들에 게 제시하고, 학생들은 10-15분 정도 소그룹 내에서 토론하 면서 문제를 푼다. 이때 교수와 조교들은 소그룹들을 옮겨 다니면서 소그룹 내에서 해결되지 않는 문제나 컨텐츠와 관 련된 질문에 답한다. 그리고 나면 교수는 학생들에게 모범 답안을 보여주며 마무리 한 후 다음 주제로 넘어간다.

주중 2 번 교실에서 만나는 동질 (같은 학력 수준) 의 2 개의 분반에 다음과 같은 차이를 두고 이 방법을 적용했다.

- 첫째, 한 분반에는 전체 강의 시간에 대해 면대면 강 의의 분량이 $1 / 2$, 다른 한 분반에는 $1 / 3$ 이 되도록 유 지했다.

- 둘째, $1 / 3$ 분반에서 오로지 소그룹 문제 풀이 중심의 강의만 진행하는 주중 두번째 수업의 참여 여부는 학생들이 스스로 선택하도록 했다. 우리는 이러한 방식으로 학습자의 자유를 강조하기로 했고, 이 점은 플립트 러닝에서 중요한 부분이다 [1].

수업 이후에 진행되는 요소들은 전통적인 물리 강의 형식과 같다. 매주 학생들에게 서술형 문제를 과제로 내주었으며,
핵심 주제와 과제 문제를 조교와 토의할 수 있는 연습반이 진행되었다.

위에 제시한 2 개 분반의 학업 성취도를 비교하기 위해, 학기별 중간고사와 기말고사 성적을 활용한다. 학기 별로 시험 문제들은 달랐으나, 같은 학기 내에서는 분반에 상관 없이 똑같은 시험 문제를 제시하였다. 두 분반 학생들의 수업 만족도를 비교하기 위해 학기 말에 설문 조사를 시행 하였다. 이 설문 조사의 내용은 다음과 같았으며, 각각의 요소는 1 점에서 5 점으로 다섯 단계의 척도로 측정했다.

- 면대면 강의 (L: Lecture) - 수업 시간에 들었던 교수 자의 강의는 얼마나 도움이 되었는가?

- 동료 학습 (PI: Peer Instruction) - 수업 시간에 동 료들과 함께 진행한 문제 풀이와 토론 시간은 얼마나 도움이 되었는가?

- 수업 설계 (ID: Instructional Design) - 플립트 러 닝을 기반으로 설계된 수업의 체계는 얼마나 도움이 되었는가?

첫째, '면대면 강의 $(\mathrm{L})^{\prime}$ 의 요소는 $1 / 2$ 분반과 $1 / 3$ 분반에 서 각각 학생들에게 면대면 강의가 얼마나 도움이 되었는가 를 측정하고자 했다. 두 분반에서 강의한 시간은 다르지만, 똑같은 교수자가 같은 내용을 강의했기 때문에, 면대면 강의 비중과 만족도 사이의 관계를 확인할 수 있다.

둘째, '동료 학습 $(\mathrm{PI})$ '의 요소는 강의의 요소를 대체한 문제 풀이 기반의 동료 학습에 대한 학생들의 만족도를 측 정하고자 했다. 일반적으로 강의를 통해 주어진 지식을 확 인하기 위한 도구로서의 문제 풀이가 아니라, 강의에서 주 어진 핵심 개념을 세워가고 정련하는 도구로서 동료 학습이 잘 동작하는지 확인하려 했다.

셋째, '수업 설계 (ID)'의 요소는 면대면 강의를 줄이고 동료 학습을 늘린 방식이 체계적으로 학습에 도움이 되는 방 향으로 동작하고 있었는지를 측정하고자 했다. 예를 들어, 어떤 학생은 면대면 강의의 분량이 줄어들어서 내용 파악이 쉽지 않았지만, 동료 학습을 통해서 그것을 잘 극복할 수 있 었다고 느낄 수 있다. 이 경우, 면대면 강의와 동료 학습에 대한 만족도를 측정하는 것만으로는 수업 구성 체계에 대한 만족도를 측정할 수는 없다.

설문 조사의 결과는 SPSS 21.0 for Windows (SPSS)를 사용하여 다음과 같이 두 단계로 통계분석을 시도하였다. 첫째, $1 / 3$ 분반과 $1 / 2$ 분반의 학업 성취도 및 수업 만족도 차 이를 알아보기 위해, 빈도분석, 기술통계 $(m, s d), t$-test를 실시하였다. 둘째, $1 / 3$ 분반과 $1 / 2$ 분반의 학업 성취도와 수 업 만족도의 관계를 확인하기 위해, 상관분석 (correlation analysis) 과 다중선형회귀분석 (multiple liner regression analysis)을 실시하였다. 
Table 2. Students' Achievements. $m$ and sd stand for mean and standard deviation, respectively. $N$ indicates the number of students in the section.

\begin{tabular}{|c|c|c|c|c|c|c|}
\hline \multirow{2}{*}{ Semester } & \multirow{2}{*}{ Section } & \multirow{2}{*}{$N$} & \multicolumn{2}{|c|}{ Midterm Exam } & \multicolumn{2}{|c|}{ Final Exam } \\
\hline & & & $m$ & $s d$ & $m$ & $s d$ \\
\hline \multirow{2}{*}{$1 \mathrm{st}$} & $1 / 3$ & 140 & 54.239 & 17.868 & 53.828 & 20.838 \\
\hline & $1 / 2$ & 151 & 54.556 & 16.830 & 57.854 & 18.171 \\
\hline \multicolumn{3}{|c|}{$P$-value } & \multicolumn{2}{|c|}{0.876} & \multicolumn{2}{|c|}{0.277} \\
\hline \multicolumn{3}{|c|}{$t$-value } & \multicolumn{2}{|c|}{-0.156} & \multicolumn{2}{|c|}{-1.088} \\
\hline \multirow{2}{*}{ 2nd } & $1 / 3$ & $\overline{156}$ & 59.740 & 19.074 & 60.365 & 21.532 \\
\hline & $1 / 2$ & 137 & 61.616 & 19.360 & 59.970 & 21.720 \\
\hline \multicolumn{3}{|c|}{$P$-value } & \multicolumn{2}{|c|}{0.405} & \multicolumn{2}{|c|}{0.876} \\
\hline \multicolumn{3}{|c|}{$t$-value } & \multicolumn{2}{|c|}{-0.834} & \multicolumn{2}{|c|}{0.156} \\
\hline
\end{tabular}

\section{III. 결 과}

\section{1. 학업 성취도 (Students' Achievements)}

Table 2 에서 학기별로 중간고사와 기말고사의 결과로 2 개 분반의 학업 성취도를 비교해보면 통계적으로 차이가 없다.

첫번째 학기는 $1 / 3$ 분반과 $1 / 2$ 분반의 중간고사 성적의 평 균은 $1 / 2$ 분반 $(54.556)$ 이 $1 / 3$ 분반 (54.239) 보다 더 높았으 나 $t=0.156$ 으로 유의수준 0.05 에서 유의한 차이가 없었다. 기말고사 성적 또한 $1 / 2$ 분반(57.854)이 $1 / 3$ 분반 (53.828) 보다 평균이 더 높았으나 $t=-1.088$ 로 유의수준 0.05 에서 유의한 차이가 없는 것으로 나타났다.

두번째 학기도 마찬가지였다. 중간고사 성적은 $1 / 2$ 분 반(61.616)이 $1 / 3$ 분반(59.740) 보다 평균이 더 높았으나 $t=-0.834$ 로 유의수준 0.05 에서 유의한 차이가 없었으며, 기말고사 성적도 $1 / 3$ 분반(60.365)이 $1 / 2$ 분반(59.970)보다 평균이 더 높았으나 $t=0.156$ 으로 유의수준 0.05 에서 유 의한 차이가 없는 것으로 나타났다.

따라서 우리가 제안한 플립트 러닝은 면대면 강의 분량 에 변화를 주더라도 학생들의 학업 성취도는 크게 다르지 않았다고 해석할 수 있다. 이것은 플립트 러닝의 장점을 지지한다. 즉, 학습의 중요한 과정은 변인이 되었던 면대면 강의에 크게 좌지우지되지 않는다. 선행 연구 [4]에서 대학 신입생을 대상으로 하는 1 학기 물리학 수업에 플립트 러닝 을 적용하여 얻은 결론과도 동일하다.

하지만, 여기에는 두 가지 주의해야 할 측면이 있다. 첫째, 우리가 살펴본 강의 분량은 $1 / 3$ 과 $1 / 2$ 정도의 수준이었다. $1 / 3$ 에서 $1 / 2$ 까지 강의 분량을 줄이더라도 학업 성취도가 다 르지 않다는 것은 우리가 설계한 플립트 러닝의 동료 학습과 여러 활동들이 잘 동작하고 있다는 것을 보여준다. 하지만 우리의 모델을 적용하여 면대면 강의 분량을 이보다 더 줄일 때에도 같은 결과를 얻을 수 있을지는 보다 세심하게 연구
해야 한다. 둘째, 이 결과는 학생들이 각 분반에서 직면해야 했던 심리적인 도전과 어려움에 대해서는 아무 것도 말하지 않는다. 실제 교실 수업을 진행하면서 학생들 사이에 강의 분량이 줄어드는 것에 대해 막연한 두려움과 걱정이 있다는 것은 감지할 수 있었다. 이제 곧 보게 되겠지만, 이것은 설 문 조사를 통해서도 확인할 수 있었던 결과였으며, 플립트 러닝을 적용하여 수업을 설계할 때 이 점을 유의해야 한다.

\section{2. 수업 만족도 (Students' Satisfaction)}

수업 만족도의 면대면 강의 $(\mathrm{L})$, 동료 학습 $(\mathrm{PI})$, 수업 설 계(ID) 의 결과를 바교하면 Table 3 와 같다. 세 가지 모든 항목에 대해 학생들의 만족도는 비교적 높았다. 그러나 항 목간을 비교해 보면, 다음과 같은 사실을 파악할 수 있다. 면대면 강의의 분량이 줄었을 때, 학생들은 동료 학습이 자 신의 학습 과정에 많은 도움이 된다고 생각하면서도, 여전 히 면대면 강의를 통해 많은 것을 배우고 있다고 생각했다. 그러나 학생들은 플립트 러닝 기반의 수업 설계보다는 면 대면 강의나 동료 학습의 요소에 보다 더 만족했던 것으로 보인다. 항목별로 수업 만족도의 차이를 살펴보면 다음과 같다.

첫째, 동료 학습의 만족도는 면대면 강의가 줄어든 분반 에서 더 높았다. 면대면 강의 만족도 $(\mathrm{L})$ 는 $1 / 3$ 분반(4.016) 이 $1 / 2$ 분반(3.895) 보다 평균이 더 높은 것으로 나타났으나, $t=1.904$ 로 유의수준 0.05 에서 유의한 차이가 없었다. 하 지만 동료 학습 만족도 $(\mathrm{PI})$ 는 $1 / 3$ 분반 (4.158)이 $1 / 2$ 분반 (4.027) 보다 평균이 더 높은 것으로 나타났으며, $t=2.016$ 으로 유의수준 0.05 에서 유의한 차이가 있었다. 따라서, 면대면 강의가 상대적으로 줄어들더라도 학생들은 동료 학 습을 통해서 그것을 보완할 수 있었다고 생각하는 것으로 보인다.

둘째, 면대면 강의가 줄어든 분반에서는 수업 설계에 대 한 불만족이 심화되었다. 수업 설계 만족도(ID) 는 $1 / 2$ 분반 (3.812)이 $1 / 3$ 분반(3.385) 보다 평균이 더 높은 것으로 나 타났으며, $t=-5.085$ 로 유의수준 0.001 에서 매우 유의한 차이가 있었다. 면대면 강의가 줄어들면, 학생들은 동료 학습을 통해서 학습 내용을 따라가는 것에 실제적인 차이는 없지만, 수업에 대해 불만족을 느끼는 것으로 보인다.

결론적으로 면대면 강의의 요소가 동료 학습의 요소로 전환되더라도 실제적인 학업 성취도는 크게 차이가 없지만, 다른 요소들보다 수업 설계에 대한 불만족이 가장 확실하게 증가하였다. 
Table 3. Students' Satisfaction for L (Lecture), PI (Peer Instruction) and ID (Instructional Design). $\mathrm{m}$ and $\mathrm{sd}$ stand for mean and standard deviation, respectively. $N$ indicates the number of students in the section including two semesters.

\begin{tabular}{c|c|cc|cc|cc}
\hline \hline \multirow{2}{*}{ Section } & \multirow{2}{*}{$N$} & \multicolumn{2}{|c|}{$\mathrm{L}$} & \multicolumn{2}{|c|}{ PI } & \multicolumn{2}{c}{ ID } \\
\cline { 3 - 7 } & & $m$ & $s d$ & $m$ & $s d$ & $m$ & $s d$ \\
\hline $1 / 3$ & 296 & 4.016 & 0.787 & 4.158 & 0.726 & 3.385 & 1.126 \\
\hline $1 / 2$ & 288 & 3.895 & 0.748 & 4.027 & 0.838 & 3.812 & 0.887 \\
\hline P-value & \multicolumn{2}{|c|}{0.057} & \multicolumn{2}{|c|}{0.044} & 0.000 \\
\hline t-value & \multicolumn{2}{|c|}{1.904} & \multicolumn{2}{|c|}{2.016} & \multicolumn{2}{|c}{-5.085} \\
\hline \hline
\end{tabular}

\section{3. 수업 만족도와 학업 성취도와 상관관계}

수업 방식의 각 요소들이 수강생의 학업 성취도에 어떠 한 영향을 미쳤는지를 면밀하게 살펴보기 위해 상관 분석 (correlation analysis) 을 실시했다. 여기서 수업 만족도를 확인하는 설문 조사의 문항들은 학기나 분반에 상관 없이 동일했다. 학업 성취도를 확인하는 중간고사와 기말고사 시험 문제는 학기별로는 달랐으나, 동일 학기내에서는 분반 에 상관없이 같았다. 따라서 다음과 같은 과정으로 분반별 학업 성취도를 새롭게 환산했다.

- 모든 학기 각 분반의 기말고사 점수를 중간고사 점수 로 표준화했다. 표본의 숫자가 충분히 많으므로 정 규 분포를 가정하고 기말 고사의 평균과 표준 편차를 중간 고사의 평균과 표준 편차가 되도록 환산했다. 각 분반에서 기말고사 점수가 중간고사 점수에 비해 얼마나 향상되었느냐를 참고하여 수업 만족도와의 상관 관계를 고려하려 하였다. (하지만 Table 2에서 보듯이 중간고사 성적과 기말고사 성적의 분포 차이가 크지 않아서 환산 점수가 크게 달라지지는 않았다.)

- 분반별로 중간고사와 기말고사 점수를 합산하여 한 학기의 학업 성취도로 정의한다.

분석 결과는 $1 / 3$ 분반의 수업 만족도와 학업 성취도 사이 에 유의한 상관 관계가 없었으나, $1 / 2$ 분반은 유의한 상관 관계가 있는 것으로 나타났다. 세부적으로 살펴보면 다음과 같다.

첫째, $1 / 3$ 분반의 수업 만족도와 학업 성취도의 상관관계 는 Table 4 에 정리되었다. 면대면 강의와 성적의 상관관 계는 $r=0.018(p>0.05)$, 동료 학습과 성적의 상관관계 는 $r=0.106(p>0.05)$, 수업 설계와 성적의 상관관계는 $r=-0.038(p>0.05)$ 으로 나타났다. 결과적으로 $1 / 3$ 분 반의 수업 만족도는 성적과 유의한 상관관계가 없는 것으로 확인되었다.
Table 4. Correlation between students' satisfaction (L, PI, ID ) and achievement (SA) in $1 / 3$ sections $(\mathrm{N}=296)$. ${ }^{*}: p<0.05,{ }^{* *}: p<0.01,{ }^{* * *}: p<0.001$.

\begin{tabular}{c|c|c|c|c}
\hline \hline & $\mathrm{L}$ & $\mathrm{PI}$ & $\mathrm{ID}$ & $\mathrm{SA}$ \\
\hline $\mathrm{L}$ & 1 & & & \\
\hline PI & $0.410^{* * *}$ & 1 & & \\
\hline ID & $0.191^{* *}$ & $0.273^{* * *}$ & 1 & \\
\hline SA & 0.018 & 0.106 & -0.038 & 1 \\
\hline \hline
\end{tabular}

Table 5. Correlation between students' satisfaction (L, PI, ID ) and achievement (SA) in $1 / 2$ sections $(\mathrm{N}=288)$.* $^{*}$ $p<0.05,{ }^{* *}: p<0.01,{ }^{* * *}: p<0.001$.

\begin{tabular}{c|c|c|c|c}
\hline \hline & $\mathrm{L}$ & $\mathrm{PI}$ & $\mathrm{ID}$ & $\mathrm{SA}$ \\
\hline $\mathrm{L}$ & 1 & & & \\
\hline PI & $0.321^{* * *}$ & 1 & & \\
\hline ID & $0.296^{* *}$ & $0.564^{* * *}$ & 1 & \\
\hline SA & $0.141^{*}$ & $0.187^{* *}$ & $0.216^{* *}$ & 1 \\
\hline \hline
\end{tabular}

둘째, $1 / 2$ 분반의 수업 만족도와 학업 성취도의 상관관계 는 Table 5 에 정리되어 있다. 면대면 강의와 성적의 상관 관계는 $r=0.141(p<0.05)$, 동료 학습과 성적의 상관관 계는 $r=0.187(p<.01)$, 수업 설계와 성적의 상관관계는 $r=0.216(p<.001)$ 로 나타났다. 결과적으로 $1 / 2$ 분반 은 수업 만족도의 모든 요소와 학업 성취도 사이에 유의한 상관관계가 있는 것으로 확인되었는데, 면대면 강의, 동료 학습, 수업 설계 순으로 상관관계가 강한 것으로 나타났다.

$1 / 3$ 분반과 $1 / 2$ 분반의 수업만족과 성적의 상관관계를 검 증한 결과, $1 / 3$ 분반의 수업만족과 성적은 유의한 상관관 계가 없었으며, $1 / 2$ 분반의 수업만족과 성적은 유의한 상관 관계가 있는 것으로 나타났다. 이 결과로부터 $1 / 3$ 분반은 그렇지 않지만, $1 / 2$ 분반은 수업의 요소들에 만족하는 학생 일수록 학업 성취도가 높았다는 것을 알 수 있다. 이를 통해 $1 / 3$ 분반보다는 $1 / 2$ 분반에 플립트 러닝을 기반으로 하는 수업이 시스템적으로 잘 동작하는 것으로 판단할 수 있다.

$1 / 2$ 분반의 수업만족이 성적에 어떠한 영향을 미치는지를 규명하기 위해 다중선형회귀분석 (multiple liner regression analysis) 을 실시하였으며, 결과는 Table 6 와 같다. 이 결과 에 따르면 플립트 러닝이 적용된 $1 / 2$ 분반의 수업 만족도 중 수업 설계만이 성적에 유의한 영향을 미치는 것으로 나타났 다. 전체만족은 성적에 긍정적 영향 $(\beta=3.149, p<0.05)$ 을 미치고 있었으며, $1 / 2$ 분반의 수업만족은 성적 전체 변량 의 $5.7 \%(F=5.733, p<0.01)$ 를 설명하고 있었다.

면대면 강의를 줄이는 플립트 러닝을 적용할 때 학생들은 수업 설계를 다른 요소들에 비해 만족하지 않는 듯 보이지 만, 바로 이 수업 설계를 긍정적으로 생각하는 학생일수록 학업 성취도가 높았다. 하지만 강의 분량이 다소 높은 $1 / 2$ 분반에서는 그런 경향성이 높지만, 강의 분량이 적은 $1 / 3$ 분반에서는 그렇지 않았다. 
Table 6. The effects of students' satisfaction (L, PI, ID) on students' achievement (SA) $(\mathrm{N}=288)$. $^{*}: \mathrm{p}<0.05,{ }^{* *}$ : $\mathrm{p}<0.01,{ }^{* * *}: \mathrm{p}<0.001$.

\begin{tabular}{|c|c|c|c|c|c|c|c|}
\hline Explanatory Variable & Dependent Variables & $\beta$ & $\mathrm{SE}$ & Beta & $\mathrm{t}$ & $\mathrm{R}^{2}$ & F-value \\
\hline $\mathrm{L}$ & \multirow[t]{4}{*}{ s } & 1.769 & 1.530 & .071 & 1.156 & \multirow{4}{*}{0.057} & \multirow{4}{*}{$5.733^{* *}$} \\
\hline $\mathrm{PI}$ & & 1.757 & 1.581 & 0.079 & 1.111 & & \\
\hline ID & & 3.149 & 1.482 & 0.150 & $2.215^{*}$ & & \\
\hline Intercept & & 34.036 & 6.863 & & $4.959^{* * *}$ & & \\
\hline
\end{tabular}

\section{IV. 결론과 토의}

플립트 러닝을 수업에 적용할 때, 우리는 무엇보다 학습 성취도가 향상되거나 적어도 유지되기를 기대한다. 그러나 그와 함께 수업 참여 학생들의 학습 과정에 점수화 되지 않 는 자기 인식과 평가에 대해서도 반드시 유의해야만 한다. 왜냐하면 교육은 점수로 파악되는 적절한 학업 성취도에 도달하는 것만이 아니라 학습자가 어떻게 성장하였는지에 대한 심리 고찰까지 포함해야 하기 때문이다.

플립트 러닝의 핵심은 전통적인 면대면 강의를 줄이는 대신, 학생들이 동료 학습 기반의 문제 풀이 및 토론 활동에 시간을 활용하도록 하는 데에 있다. 그러나 다른 기법들 을 시도하기 위해 면대면 강의를 얼마나 줄일 수 있는지는 과목과 주제에 따라 다르다. 예컨대, 고급 물리학 과목의 몇몇 주제들은 복잡한 수식을 전개하여 긴 호흡의 설명을 해야만 한다. 따라서 면대면 강의의 분량을 정하는 것은 매우 복잡하고 정교한 교수 학습 작업이다.

본 논문에서 우리는 대학 신입생들이 두번째 학기에 배우 는 기초 물리학 과목인 일반 물리 2 에 플립트 러닝을 적용할 때 면대면 강의 분량의 역할을 살펴보았다. 우리는 $1 / 3$ 과 $1 / 2$ 의 제한된 범위 안에서 면대면 강의 분량을 조절하면서, 수강생들의 학업 성취도, 수업 만족도 그리고 그들 사이의 상관 관계를 2 개 학기 동안 총 4 개반의 분반을 대상으로 조사하였다. 이러한 범위 안에서 중간고사와 기말고사 성 적으로 나타낼 수 있는 두 분반의 학업 성취도는 통계적으로 다르지 않았으며, 면대면 강의, 동료 학습, 수업 설계의 세 부분으로 이뤼진 수업 만족도도 두 분반 모두 전반적으로 높았다. 그러나 두 분반 사이에 가장 명확한적 차이점은 바로 수업 설계에 대한 만족도였다. 첫째, $1 / 3$ 분반에 비해 $1 / 2$ 분반은 수업 설계에 대한 만족도가 높았다. 둘째, $1 / 3$ 분반은 그렇지 않으나 $1 / 2$ 분반은 수강생의 학업 성취도와 수업 만족도 사이에 상관관계가 나타났다. 즉, 학업 성취 도가 높은 학생일수록 플립트 러닝으로 설계된 수업 체계 에 만족하고 있는 것으로 보인다. 종합적으로 보면 수업이 설계된 방식을 즐겁게 따라가며 공부한 학생들이 성공할 수 있었다는 점에서 $1 / 3$ 분반 보다는 $1 / 2$ 분반에서 플립트 러닝이 체계적으로 동작했다고 판단할 수 있었다.
이것은 우리에게 적어도 다음과 같은 두 가지 의미를 갖 는다. 첫째, 플립트 러닝을 적용하는 수업에서 전통적인 면대면 강의의 비중을 세심하게 정해야 한다는 점이다. 학 생들은 여전히 스토링텔링 능력이 뛰어난 교수에게서 아름 답고 매끄럽게 준비된 강의를 들을 때 보다 긍정적인 학습 경험을 한다고 생각한다. 다소 주관적이기는 하지만, 이것 은 수업의 질적 요소로서 학생들이 학습 경험을 형성하는 데 있어서 결코 무시할 수 없는 심리적인 부분이다. 이러한 요소가 부족하다면, 비록 최종적인 학업 성취도에는 큰 차 이가 없을지라도, 학생들은 학습 과정에 대해 불만족하게 된다. 둘째, 학생들이 수업을 평가할 때 면대면 강의나 동료 학습과 같은 개별적 요소에만 영향을 받는 것이 아니라는 점이다. 어떤 한 가지 요소가 부족하더라도 다른 요소들이 그것을 보완하여 보다 효과적인 수업이 될 수 있도록 교수 학습 설계의 구조적인 부분에 대해 세심한 고려를 해야한다.

오늘날 우리는 COVID-19의 세계적 대유행으로 인해 대 학의 수업이 비대면으로 진행될 수 밖에 없는 전대미문의 시대에 살고 있다. 이것은 학습자에게 뿐만 아니라 교수자 에게도 매우 도전적인 과제를 남겨준다. 비대면 강의를 통 해 강의의 내용을 전달하는 것이 쉽지 않다면, 학습 효과를 높이기 위해 동영상과 과제의 수를 늘려가는 방식으로만 이 문제에 효과적으로 대처할 수 있을까? 학습자들이 감내 해야하는 스트레스가 있는데, 이런 방식으로 배우는 교과 목들이 늘어간다면, 학생들은 정말 잘 배울 수 있겠는가? 우리의 연구는 교수 학습 설계를 학습자의 환경과 학습자의 반응에 근거해 매우 세심하게 진행해야 할 것을 함의하고 있다.

\section{감사의 글}

저자들은 포항공대의 임진혁 교수님, UNIST 의 이해웅 교수님, 신국조 교수님, 진근영 박사님, 범수균 박사님, 서 윤영 선생님, DGIST의 이기준 교수님, 최승호 교수님, 박 기성 교수님께 감사드립니다. 


\section{REFERENCES}

[1] J. Bergmann and A. Sams, Flip Your Classroom: Reach Every Student in Every Class Every Day, International Society for Technology in Education (2012).

[2] J. Bergmann and A. Sams, Flipped Learning: Gateway to Student Engagement, International Society for Technology in Education (2014).

[3] S. Bates and R. Galloway, "The inverted classroom in a large enrolment introductory physics course: a case study," Proceedings of the HEA STEM Learning and Teaching Conference (2012).

[4] H. Zoe et al., "Role of face-to-face lecturing in large enrollment physics classes," arXiv:1404.3566 [physics.ed-ph]

[5] G. M. Novak, E. T. Patterson, A. D. Gavrin and W. Christian, Just in Time Teaching, Prentice Hall, Upper Saddle River, NJ (1999).

[6] E. Mazur, Peer Instruction: A User's Manual, Prentice Hall, Upper Saddle River, NJ (1997).

[7] C. Crouch and E. Mazur, Am. J. Phys. 69, 970 (2001).

[8] Z. Chen, T. Stelzer and G. Gladding, Phys. Rev. ST Phys. Educ. Res. 6, 010108 (2010).

[9] K. O'Connor, Higher Education, 68, 623 (2014).

[10] J. Wulf, I. Blohm, J. M. Leimeister, and W. Brenner, Business \& Information Sys. Egin., 6, 111 (2014).

[11] P. Heller, R. Keith, and S. Anderson, Am. J. Phys. 60, 627 (1992).

[12] P. Heller and M. Hollabaugh, Am. J. Phys. 60, 637 (1992).

[13] R. Hake, Am. J. Phys. 66, 64 (1998).

[14] Y. J. Dori, E. Hult, L. Breslow and J. W. Belcher, J. Sci. Educ. Technol. 16, 299 (2007).
[15] M. Smith et al., Science, Vol. 323, no. 5910 pp. 122 (2009).

[16] L. Deslauiers, E. Schelew and C. Wieman, Science, Vol. 332 no. 6031 pp. 862 (2011)

[17] H. Berger, B. Eylon and F. Bagno, J. Sci. Educ. Technol. 17, 399(2008).

[18] F. Goldberg et al., Phys. Rev. ST Phys. Educ. Res. 8, 010121 (2012).

[19] Jonassen, D. (1999) Designing constructivist learning environments, In C. Reigeluth (Ed.), Instructional design theories and models (Vol. 2). Mahwah, NJ: Lawrence Erlbaum.

[20] L. McDermott and E. Redish, Am. J. Phys. 67, 755 (1999).

[21] D. Meltzer and R. Thornton, Am. J. Phys. 80, 478 (2012).

[22] S. Pollock and N. Finkelstein, AIP Conf. Proc. 1513, 31 (2013)

[23] W. Potter, C. DeLeone and L. Coleman, AIP Conf. Proc. 399, 829 (1996).

[24] E. Redish, Teaching Physics with the Physics Suite, John Wiley \& Sons Inc. (2003)

[25] A. Rudolph et al., Phys. Rev. ST Phys. Educ. Res. 10, 010103 (2014)

[26] D. G. Sampson, D. Ifenthaler, J. M. Spector, and P. Isaias, Digital system for open access to formal and informal learning, Springer (2014).

[27] T. Stelzer, G. Gladding, and J. Mestre, Am. J. Phys. 78, 755 (2010).

[28] E. West, C. Paul, D. Webb and W. Potter, Phys. Rev. ST Phys. Educ. Res. 9, 010109 (2013). 